Honam Mathematical J. 32 (2010), No. 4, pp. 769-776

\title{
PEBBLING EXPONENTS OF PATHS
}

\author{
Ju Young Kim And Sun Ah Kim
}

\begin{abstract}
A pebbling move on a connected graph $G$ is taking two pebbles off of one vertex and placing one of them on an adjacent vertex. For a connected graph $G, G^{p}(p>1)$ is the graph obtained from $G$ by adding the edges $(u, v)$ to $G$ whenever $2 \leq \operatorname{dist}(u, v) \leq p$ in $G$. And the pebbling exponent of a graph $G$ to be the least power of $p$ such that the pebbling number of $G^{p}$ is equal to the number of vertices of $G$. We compute the pebbling number of fourth power of paths so that the pebbling exponents of some paths are calculated.
\end{abstract}

\section{Introduction}

Pebbling in graphs was first considered by Chung[1]. Consider a connected graph with a fixed number of pebbles distributed on its vertices. A pebbling move consists of removing two pebbles from one vertex $u$ and then placing one pebble at an adjacent vertex $v$. We say that we can pebble to a vertex $v$, the target vertex, if we can apply pebbling moves repeatedly so that it is possible to reach a configuration with at least one pebble at $v$. The pebbling number of a vertex $v$ for a graph $G$, denoted by $f(G, v)$, is the smallest integer $m$ which guarantees that any starting pebble configuration with $m$ pebbles allows pebbling to $v$. And the pebbling number of $G$, denoted by $f(G)$, as the maximum of $f(G, v)$, over all vertices $v$.

A graph $G$ is called demonic if $f(G)$ is equal to the number of its vertices. If one pebble is placed on each vertex other than the vertex $v$, then no pebble can be moved to $v$. Also, if $w$ is at distance $d$ from $v$, and $2^{d}-1$ pebbles are placed on $w$, then no pebble can be moved to $v$. So it is clear [1] that $f(G) \geq \max \left\{|V(G)|, 2^{D}\right\}$, where $|V(G)|$ is the number of the vertices of $G$ and $D$ is the diameter of $G$. Let $G=(V(G), E(G))$ be a connected graph. Then $G^{p}(p>1)$ (the $p$ th power of $G$ ) is the graph obtained from $G$ by adding the edges $(u, v)$ to $G$ whenever $2 \leq \operatorname{dist}(u, v) \leq p$ in $G$. Hence $G^{p}=(V(G), E(G) \cup F(G))$

Received November 19, 2010. Accepted December 11, 2010.

Key words and phrases: exponent, path, pebbling. 
where $F(G)=\{(u, v): 2 \leq \operatorname{dist}(u, v) \leq p$ in $G\}$. If $p=1, G^{1}=G$. In [5], they proved $f\left(P_{2 k+r}{ }^{2}\right)=2 k+r$ where $o \leq r \leq 1$. Let $|V(G)|=n$. We know that if $p$ is large enough (i.e., $p \geq n-1$ ) then $G^{p}=K_{n}$.

In [6], the pebbling exponent of a graph $G$ (denoted by $e(G)$ ) is defined to be the least power of $p$ such that $f\left(G^{p}\right)=|V(G)|$.

In section 2 , we calculate the pebbling number of the fourth power of paths. Using the results in section 2, we give the pebbling exponent of some paths in section 3 .

\section{The pebbling number of fourth power of $P_{n}$}

In [6], $f\left(P_{n}{ }^{2}\right)$ was calculated but the proof is too long. So that result was reproved by more simple method in [7]. It is known that the pebbling number $f\left(P_{n}\right)$ of the path $P_{n}$ with $n$ vertices is $2^{n-1}[1] . p(v)$ will denote the number of pebbles at vertex $v$.

In [7], the following two lemmas were used to calculate $f\left(P_{n}{ }^{2}\right)$ and $f\left(P_{n}^{3}\right)$.

Lemma 1. [7] Let $P_{n}{ }^{2}=x_{1} x_{2} \cdots x_{n-1} x_{n}$ with $n \geq 7$. If $p(x)$ is even for each vertex $x$ of the graph $P_{n}^{2}$, then $2^{\left\lceil\frac{n-1}{2}\right\rceil}$ pebbles are sufficient to pebble $x_{1}$ or $x_{n}$.

Lemma 2. [7] Let $P_{n}{ }^{3}=x_{1} \cdots x_{n}$ with $n \geq 8$. If $p(x)$ is even for each vertex $x$ of $P_{n}^{3}$, then $2^{\left\lceil\frac{n-1}{3}\right\rceil}$ pebbles are sufficient to pebble $x_{1}$ or $x_{n}$.

Similarly we can get the following lemma 3 .

Lemma 3. Let $P_{n}{ }^{k}=x_{1} \cdots x_{n}$ with $n \geq 2 k \geq 8$. If $p\left(x_{i}\right)$ is even for each vertex $x_{i}$ of $P_{n}{ }^{k}$, then $2^{\left\lceil\frac{\lceil-1}{k}\right\rceil}$ pebbles are sufficient to pebble $x_{1}$ or $x_{n}$.

Proof. Let $P_{n}{ }^{k}=x_{1} x_{2} \cdots x_{n}$ with $8 \leq 2 k \leq n, p\left(x_{i}\right)$ be even for $x_{i}$. Place $2^{\left\lceil\frac{n-1}{k}\right\rceil}$ pebbles on $P_{n}{ }^{k}$. By symmetry, we assume that $v=x_{1}$. Let $p\left(x_{1}\right)=0$. If $\left\lceil\frac{n-1}{k}\right\rceil=t$, we can write $P_{n}{ }^{k}$ as $P_{n}{ }^{k}=$ $x_{0 k+1} x_{0 k+2} \cdots x_{1 k} x_{1 k+1} \cdots x_{1 k+k} x_{2 k+1} \cdots x_{(t-1) k} x_{(t-1) k+1} \cdots x_{n}$ where $(t-$ $1) k+1<n \leq t k+1$. By moving as many pebbles as possible from each $x_{j k+r}(2 \leq r \leq k+1)$ to $x_{j k+1}$ for $j=0,1, \cdots,(t-1)$, there can be at least $2^{t-1}$ pebbles at the vertices $x_{0 k+1} x_{1 k} \cdots x_{(t-1) k+1}$. Since $x_{0 k+1} x_{1 k+1} \cdots x_{(t-1) k+1}$ is isomorphic to $P_{t}$ and $f\left(P_{t}\right)=2^{t-1}$, we can put a pebble at $x_{1}$.

Theorem 4. [6] $f\left(P_{2 k+r}{ }^{2}\right)=2^{k}+r$ when $0 \leq r \leq 1$. 
Theorem 5. [7] $f\left(P_{n}{ }^{3}\right)=n$ if $1 \leq n \leq 7$. For $n \geq 8$,

$$
f\left(P_{n}^{3}\right)=\left\{\begin{array}{rlll}
2^{\left\lfloor\frac{n}{3}\right\rfloor}+1 & \text { if } & n \equiv 0 & (\bmod 3) \\
2^{\left\lfloor\frac{n}{3}\right\rfloor}+2 & \text { if } & n \equiv 1 & (\bmod 3) \\
2^{\left\lfloor\frac{n}{3}\right\rfloor+1} & \text { if } & n \equiv 2 & (\bmod 3)
\end{array}\right.
$$

Above two theorems can be restated as followings.

Theorem $4^{\prime} . f\left(P_{n}{ }^{2}\right)=2^{\left\lceil\frac{n-1}{2}\right\rceil}+r \quad$ when $\quad 0 \leq r \leq 1$

Theorem $\mathbf{5}^{\prime} . f\left(P_{n}^{3}\right)=2^{\left\lceil\frac{n-1}{3}\right\rceil}+r$ when $0 \leq r \leq 2$,

$$
n \geq 8 \text { and } n-2 \equiv r(\bmod 3)
$$

First, we note that $f\left(P_{n}{ }^{4}\right)=n$ for $1 \leq n \leq 13$. The reason for that is as follows. We proceed by induction on $n$. Clearly, the result is correct if $1 \leq n \leq 5$. Suppose that for all $n^{\prime}$ with $5 \leq n^{\prime}<n \leq 13$ we have $f\left(P_{n^{\prime}}{ }^{4}\right)=n^{\prime}$. We will show that $f\left(P_{n}{ }^{4}\right)=n$. Place $n$ pebbles at the vertices of $P_{n}{ }^{4}=x_{1} x_{2} \cdots x_{n}$ (the edges between $x_{i}$ and $x_{i+4}$ are implied for $(1 \leq i \leq 9)$ and assume first that $v \neq x_{1}$ or $x_{n}$. Let $v=x_{i}$ with $2 \leq i \leq n-1$. We see that if the subgraph $x_{1} \cdots x_{i} \equiv P_{i}^{4}$ contains at least $i$ pebbles and so we are done by induction. Otherwise the subgraph $x_{i} \cdots x_{n} \equiv P_{n-i-1}{ }^{4}$ contains at least $(n-i-1)$ pebbles and so we are done by induction. Therefore, we may assume that $v=x_{1}$ or $x_{n}$. By symmetry, we may assume that $v=x_{1}$. Suppose that $\Sigma_{i=2}^{5} p\left(x_{i}\right) \geq 1$. Let $p\left(x_{i}\right) \geq 1$ for some $2 \leq i \leq 5$. Then we can put one more pebble at $x_{i}$ by using the remaining $(n-1)$ pebbles on the subgraph $x_{2} \cdots x_{n} \equiv P_{n-1} 4$ by induction. Since $\operatorname{dist}\left(x_{1}, x_{i}\right)=1$, we are done by moving a pebble at $x_{1}$ from $x_{i}$. Otherwise $\Sigma_{i=2}^{5} p\left(x_{i}\right)=0$. Then $\sum_{i=6}^{n} p\left(x_{i}\right)=n$. For $6 \leq n \leq 9$ there are at least two pairs on the subgraph $x_{6} \cdots x_{n}$. Using these two pairs on that subgraph we can put two pebbles at $x_{5}$ and so we are done because $\operatorname{dist}\left(x_{1}, x_{5}\right)=1$. For $10 \leq n \leq 13$ the subgraph $x_{6} \cdots x_{n}$ contains at least 4 pairs which are used to put two pebbles at $x_{5}$. And we are done.

Next, we show that $f\left(P_{14}{ }^{4}\right)=2^{4}, f\left(P_{15}{ }^{4}\right)=2^{4}+1, f\left(P_{16}{ }^{4}\right)=2^{4}+2$ and $f\left(P_{17}^{4}\right)=2^{4}+3$.

(a) We show that $f\left(P_{14}^{4}\right)=2^{4}$.

First, we will show that $f\left(P_{14}^{4}\right) \geq 2^{4}$. Let $P_{14}^{4}=x_{1} x_{2} \cdots x_{14}$ (the edges between $x_{i}$ and $x_{i+4}$ are implied for $\left.1 \leq i \leq 10\right)$ and place $\left(2^{4}-1\right)$ pebbles at $x_{14}$. Then no pebble can be moved to $x_{1}$ because $\operatorname{dist}\left(x_{1}, x_{14}\right)=4$, therefore $f\left(P_{14}{ }^{4}\right) \geq 2^{4}$. Place $2^{4}$ pebbles at 
the vertices of $P_{14}{ }^{4}=x_{1} x_{2} \cdots x_{14}$. By symmetry, we may assume that our target vertex is $v=x_{1}, x_{2}, \cdots, x_{8}$, or $x_{9}$. If $\Sigma_{i=1}^{9} p\left(x_{i}\right) \geq 9$, then we are done because the subgraph $x_{1} \cdots x_{9}$ is isomorphic to $P_{9}{ }^{4}$ and $f\left(P_{9}{ }^{4}\right)=9$. If $\Sigma_{i=1}^{9} p\left(x_{i}\right)=8$, then $\Sigma_{j=10}^{14} p\left(x_{j}\right)=8$. By moving as many pebbles as possible from $x_{10}, x_{11}, \cdots, x_{13}$ or $x_{14}$ to $x_{9}$, we see that the subgraph $x_{1} \cdots x_{9} \equiv P_{9}{ }^{4}$ contains at least 9 pebbles. So we are done. If $\Sigma_{i=1}^{9} p\left(x_{i}\right) \leq 7$, then $\Sigma_{j=10}^{14} p\left(x_{j}\right) \geq 9$ and there are at least 4 pairs on the subgraph $x_{9} \cdots x_{14} \equiv P_{6}{ }^{4}$. By Lemma 3 , We can put 4 pebbles at $x_{9}$ by moving 4 pairs on the subgraph $x_{9} \cdots x_{14} \equiv P_{6}{ }^{4}$ to $x_{9}$. So we are done because $\operatorname{dist}\left(x_{k}, x_{9}\right) \leq 2$ for $1 \leq k \leq 9$.

(b) We show that $f\left(P_{15}^{4}\right)=2^{4}+1$.

First, we will show that $f\left(P_{15}{ }^{4}\right) \geq 2^{4}+1$. Let $P_{15}{ }^{4}=x_{1} x_{2} \cdots x_{15}$ (the edges between $x_{i}$ and $x_{i+4}$ are implied for $\left.1 \leq i \leq 11\right)$ and place $\left(2^{4}-1\right)$ pebbles at $x_{15}$ and one pebble at $x_{14}$. Then no pebble can be moved to $x_{1}$. Place $\left(2^{4}+1\right)$ pebbles at the vertices of $P_{15}{ }^{4}=x_{1} x_{2} \cdots x_{15}$. By symmetry, we may assume that our target vertex is $v=x_{1}, x_{2}, \cdots, x_{8}$, or $x_{9}$. If $\Sigma_{i=1}^{9} p\left(x_{i}\right) \geq 9$, then we are done because the subgraph $x_{1} \cdots x_{9}$ is isomorphic to $P_{9}{ }^{4}$ and $f\left(P_{9}{ }^{4}\right)=9$. If $\Sigma_{i=1}^{9} p\left(x_{i}\right)=8$, then $\Sigma_{j=1}^{15} p\left(x_{j}\right)=9$. By moving as many pebbles as possible from $x_{10}, \cdots, x_{14}$ or $x_{15}$ to $x_{9}$, we see that the subgraph $x_{1} \cdots x_{9} \equiv P_{9}{ }^{4}$ contains at least 9 pebbles and we are done. If $\Sigma_{i=1}^{9} p\left(x_{i}\right) \leq 7$, then $\sum_{j=10}^{15} p\left(x_{j}\right) \geq 10$ and there are at least 4 pairs on the subgraph $x_{9} \cdots x_{15} \equiv P_{7}^{4}$. By Lemma 3 , we can put 4 pebbles at $x_{9}$ by moving 4 pairs on the subgraph $x_{9} \cdots x_{15} \equiv P_{7}^{4}$. So we are done because $\operatorname{dist}\left(x_{k}, x_{9}\right) \leq 2$ for $1 \leq k \leq 9$.

(c) The proof of $f\left(P_{16}{ }^{4}\right)=2^{4}+2$ or $f\left(P_{17}{ }^{4}\right)=2^{4}+3$ are similar to those of $f\left(P_{14}^{4}\right)=2^{4}$ or $f\left(P_{15}^{4}\right)=2^{4}+1$.

Theorem 6. For $n \geq 14, f\left(P_{n}{ }^{4}\right)=2^{\left\lceil\frac{n-1}{4}\right\rceil}+r$ where $0 \leq r \leq 3$ and $n-2 \equiv r(\bmod 4)$.

Proof. First, we will show that $f\left(P_{n}{ }^{4}\right) \geq 2^{\left\lceil\frac{n-1}{4}\right\rceil}+3$ for $n-2 \equiv 3$ $(\bmod 4)$. Let $P_{n}{ }^{4}=x_{1} x_{2} \cdots x_{n}$ (the edges between $x_{i}$ and $x_{i+4}$ are implied for $1 \leq i \leq n-4)$ and place $\left(2^{\left\lceil\frac{n-1}{4}\right\rceil}-1\right)$ pebbles at $x_{n}$ and one pebble at each $x_{j}$ with $j=n-3, n-2$, and $n-1$. It is easy to see that a pebble can not be moved to $x_{1}$, therefore $f\left(P_{n}{ }^{4}\right) \geq 2^{\left\lceil\frac{n-1}{4}\right\rceil}+3$.

Since the diameter of $P_{n}{ }^{4}$ is $\left\lceil\frac{n-1}{4}\right\rceil$ for $n-2 \equiv 0(\bmod 4)$, we have $f\left(P_{n}^{4}\right) \geq 2^{\left\lceil\frac{n-1}{4}\right\rceil}$.

Similarly, $f\left(P_{n}^{4}\right) \geq 2^{\left\lceil\frac{n-1}{4}\right\rceil}+r$ for $n-2 \equiv r(\bmod 4), r=1$ or 2 . 
We proceed by induction on $n$. We have already showed that our theorem is correct if $n=14,15,16$, or 17 .

Case (a) $r=0$.

Suppose that for all $n^{\prime}$ with $n^{\prime}<n$ and $n^{\prime}-2 \equiv 0(\bmod 4)$ we have $f\left(P_{n^{\prime}}{ }^{4}\right)=2^{\left\lceil\frac{n^{\prime}-1}{4}\right\rceil}$. We will show that $f\left(P_{n}{ }^{4}\right)=2^{\left\lceil\frac{n-1}{4}\right\rceil}$. Let $n=4 l+2$ for some $l \geq 4$. Then $\left\lceil\frac{n-1}{4}\right\rceil=l+1$. Place $2^{\left\lceil\frac{n-1}{4}\right\rceil}\left(=2^{l+1}\right)$ pebbles at the vertices of $P_{n}{ }^{4}=x_{1} x_{2} \cdots x_{n}$. Let $v$ be the target vertex. Then there are the following three possible cases (a.1), (a.2) and (a.3).

(a.1) $v \neq x_{1}, \cdots, x_{4}, x_{n-3}, \cdots, x_{n-1}$ or $x_{n}$.

Let $P_{A}$ be the subgraph $x_{5} \cdots x_{n}$ and $P_{B}$ be the subgraph $x_{1} \cdots x_{n-4}$. Then both $P_{A}$ and $P_{B}$ are isomorphic to $P_{n-4}{ }^{4}$. It is easy to see that $P_{A}$ or $P_{B}$ contains at least $2^{\left\lceil\frac{(n-4)-1}{4}\right\rceil}$ pebbles. By induction we are done.

(a.2) $v=x_{1}$ or $x_{n}$.

By symmetry we assume that $v=x_{1}$. Suppose that $\Sigma_{i=2}^{5} p\left(x_{i}\right)=0$. Then there are $2^{l+1}$ pebbles on the subgraph $x_{5} \cdots x_{n}$ which is isomorphic to $P_{n-4}{ }^{4}$. By induction we can put two pebbles at $x_{5}$ using $2 \cdot 2^{l}$ pebbles on the subgraph $x_{5} \cdots x_{n}$. Because $\operatorname{dist}\left(x_{1}, x_{4}\right)=1$, we are done. Otherwise $\Sigma_{i=2}^{4} p\left(x_{i}\right) \geq 1$. Let $j=\min _{2 \leq i \leq 5}\left\{i \mid p\left(x_{i}\right) \geq 1\right\}$. For $p\left(x_{j}\right) \geq 2$, we are done. Let $p\left(x_{j}\right)=1$. Then there are $2^{l-1}$ pairs on the subgraph $x_{j} \cdots x_{n}$ because $\left\lceil\frac{\left(2^{l+1}-(n-j+1)\right.}{2}\right\rceil \geq 2^{l}=2 \cdot 2^{l-1}$. By using these $2^{l-1}$ pairs on that subgraph we can put one more pebble at $x_{j}$ and we are done.

(a.3) $v=x_{2}, x_{3}, x_{4}, x_{n-3}, x_{n-2}$, or $x_{n-1}$.

By symmetry we assume that $v=x_{k}$ for $2 \leq k \leq 4$. If $p\left(x_{1}\right) \geq 2$, then we can put a pebble at $x_{k}$ because $\operatorname{dist}\left(x_{1}, x_{k}\right)=1$. Otherwise $p\left(x_{1}\right) \leq 1$ and then $\sum_{j=2}^{n} p\left(x_{j}\right) \geq 2^{l+1}-1$. Since $\left(2^{l+1}-1\right)-(n-1)=$ $\left(2^{l+1}-1\right)-(4 l+1)=2^{l+1}-4 l-2 \geq 2^{l-1}$ for $l \geq 4$, there are $2^{l-2}$ pairs on the subgraph $x_{2} \cdots x_{n} \equiv P_{n-1}{ }^{4}$. By Lemma 3 , we can put a pebble at $x_{k}$ by using $2^{l-2}$ pairs on the subgraph $x_{2} \cdots x_{n}$ with $2^{\left\lceil\frac{(n-1)-1}{4}\right\rceil}=l$.

Case (b) $r=1$.

Suppose that for all $n^{\prime}$ with $n^{\prime}<n$ and $n^{\prime}-2 \equiv 1(\bmod 4)$ we have $f\left(P_{n^{\prime}}{ }^{4}\right)=2^{\left\lceil\frac{n^{\prime}-1}{4}\right\rceil}+1$. We will show that $f\left(P_{n}{ }^{4}\right)=2^{\left\lceil\frac{n-1}{4}\right\rceil}+1$. Let $n=4 l+3$ for some $l \geq 4$. Then $\left\lceil\frac{n-1}{4}\right\rceil=l+1$. Place $2^{\left\lceil\frac{n-1}{4}\right\rceil}+1\left(=2^{l+1}+1\right)$ pebbles at the vertices of $P_{n}{ }^{4}=x_{1} x_{2} \cdots x_{n}$. Let $v$ be the target vertex. Then there are the following three possible cases (b.1), (b.2) and (b.3).

(b.1) $v \neq x_{1}, \cdots, x_{4}, x_{n-3}, \cdots, x_{n-1}$ or $x_{n}$.

This case can be proved by the same way to (a.1).

(b.2) $v=x_{1}$ or $x_{n}$. 
By symmetry we assume that $v=x_{1}$. Suppose that $\Sigma_{i=2}^{4} p\left(x_{i}\right)=0$. Then there are $\left(2^{l+1}+1\right)$ pebbles on the subgraph $x_{5} \cdots x_{n}$ which is isomorphic to $P_{n-4}{ }^{4}$. Since $\left(2^{l+1}+1\right)-(n-4)=\left(2^{l+1}+1\right)-(4 l-1) \geq 2^{l}$ for $l \geq 4$, there are $2^{l-1}$ pairs on the subgraph $x_{5} \cdots x_{n}$ and so we can put a pebble at $x_{5}$ using these $2^{l-1}$ pairs on that subgraph with $\left\lceil\frac{(n-4)-1}{4}\right\rceil=l$ by Lemma 3 . And we put one more pebble at $x_{5}$ using the remaining $\left(2^{l}+1\right)$ pebbles on the subgraph $x_{5} \cdots x_{n} \equiv P_{n-4}{ }^{4}$ by induction. Since $\operatorname{dist}\left(x_{1}, x_{5}\right)=1$, we can put a pebble at $x_{1}$.

(b.3) $v=x_{2}, x_{3}, x_{4}, x_{n-2}, x_{n-1}$, or $x_{n}$.

By symmetry we assume that $v=x_{k}$ for $2 \leq k \leq 4$. If $p\left(x_{1}\right) \geq 2$, then we can put a pebble at $x_{k}$ because of $\operatorname{dist}\left(x_{1}, x_{k}\right)=1$. Otherwise $p\left(x_{1}\right) \leq 1$. Then there are at least $2^{l+1}$ pebbles on the subgraph $x_{2} \cdots x_{n}$ which is isomorphic to $P_{n-1} 4$. Then by Case (a) we can put a pebble at $x_{k}$.

Case (c) $r=2$.

Suppose that for all $n^{\prime}$ with $n^{\prime}<n$ and $n^{\prime}-2 \equiv 2(\bmod 4)$ we have $f\left(P_{n^{\prime}}{ }^{4}\right)=2^{\left\lceil\frac{n^{\prime}-1}{4}\right\rceil}+2$. We will show that $f\left(P_{n}^{4}\right)=2^{\left\lceil\frac{n-1}{4}\right\rceil}+2$. Let $n=4 l+4$ for some $l \geq 4$. Then $\left\lceil\frac{n-1}{4}\right\rceil=l+1$. Place $2^{\left\lceil\frac{n-1}{4}\right\rceil}+2\left(=2^{l+1}+2\right)$ pebbles at the vertices of $P_{n}{ }^{4}=x_{1} x_{2} \cdots x_{n}$. Let $v$ be the target vertex. Then there are the following three possible cases (c.1), (c.2) and (c.3).

(c.1) $v \neq x_{1}, \cdots, x_{4}, x_{n-3}, \cdots, x_{n-1}$ or $x_{n}$.

This case can be proved by the same way to (a.1)

(c.2) $v=x_{1}$ or $x_{n}$.

By symmetry we assume that $v=x_{1}$. If $\Sigma_{i=2}^{4} p\left(x_{i}\right)=0$, then there are $\left(2^{l+1}+2\right)$ pebbles on the subgraph $x_{5} \cdots x_{n}$ which is isomorphic to $P_{n-4} 4$. Since $\left(2^{l+1}+2\right)-(n-4) \geq 2^{l}$ for $l \geq 4$, there are $2^{l-1}$ pairs on the subgraph $x_{5} \cdots x_{n}$ and so we can put a pebble at $x_{5}$ using these $2^{l-1}$ pairs on that subgraph with $\left\lceil\frac{(n-4)-1}{4}\right\rceil=l$ by Lemma 3 . And we put one more pebble at $x_{5}$ using the remaining $\left(2^{l}+2\right)$ pebbles on the subgraph $x_{5} \cdots x_{n} \equiv P_{n-4}{ }^{4}$ by induction. Since $\operatorname{dist}\left(x_{1}, x_{5}\right)=1$, we can put a pebble at $x_{1}$.

(c.3) $v=x_{2}, x_{3}, x_{4}, x_{n-3}, x_{n-2}$, or $x_{n-1}$.

By symmetry we assume that $v=x_{k}$ for $2 \leq k \leq 4$. If $\Sigma_{j=1}^{3} p\left(x_{j}\right) \geq 4$, then we are done. Otherwise $\Sigma_{j=1}^{3} p\left(x_{j}\right) \leq 3$ and $\Sigma_{j=5}^{n} p\left(x_{j}\right) \geq\left(2^{l+1}+\right.$ $2)-3\left(=2^{l+1}-1\right)$. Since $\left\lceil\frac{\left(2^{l+1}-1\right)-(n-4)}{2}\right\rceil \geq 2^{l}$ for $l \geq 4$, there are $2^{l-1}$ pairs on the subgraph $x_{5} \cdots x_{n} \equiv P_{n-4}{ }^{4}$ with $\left\lceil\frac{(n-4)-1}{4}\right\rceil=l$ and so we can put a pebble at $x_{5}$ using these $2^{l-1}$ pairs on that subgraph by Lemma 3 . And we can put one more pebble at $x_{5}$ using the remaining 
$\left(2^{l}+2\right)$ pebbles on the subgraph $x_{5} \cdots x_{n} \equiv P_{n-4}{ }^{4}$ by induction. Since $\operatorname{dist}\left(x_{k}, x_{5}\right)=1$ for $2 \leq k \leq 4$, we are done.

Case (d) $r=3$.

The proof is similar to Case (c).

\section{The exponents of Paths}

Using Theorem 6 we can get the following Theorem 7 .

Theorem 7.

$$
e\left(P_{n}\right)=\left\{\begin{array}{lll}
2 & \text { if } & n \leq 6 \\
3 & \text { if } & 7 \leq n \leq 10 \\
4 & \text { if } & 11 \leq n \leq 13 \\
5 & \text { if } & 14 \leq n \leq 21
\end{array}\right.
$$

For $2 \leq k \leq n$, consider the set

$$
\begin{gathered}
E=\left\{k \mid 2^{\left\lceil\frac{n-1}{k}\right\rceil}+r \leq n \quad \text { where } 0 \leq r \leq k-1\right. \\
\text { and } n-2 \equiv r \quad(\bmod k)\}
\end{gathered}
$$

Conjecture. $e\left(P_{n}\right)=\min E$.

\section{References}

1. F.R.K.Chung, Pebbling in hypercubes, SIAM J. Disc. Math. Vol.2, No. 4(1989), pp 467-472.

2. D. Duffus and I. Rival, Graphs orientalbe as distributive lattices, Proc. Amer. Math. Soc. 88(1983), pp 197-200.

3. P. Lemke and D. Kleitman, An additional theorem on the intergers modulo n, J. Number Theory 31(1989), pp 335-345.

4. D. Moews, Pebbling graphs, J. of combinatorial Theory(Series B) 55(1992), pp 224-252.

5. H.S.Snevily and J. Foster. The 2-pebbling property and a Conjecture of Graham's, preprint.

6. L. Pachter, H.S.Snevily and B. Voxman, On pebbling graphs, Congr Number, 107(1995), pp 65-80.

7. Ju Young Kim, Pebbling exponents of graphs, J. of Natural Sciences In Catholic Univ. of Daegu, Vol.2, No. 1(2004), pp 1-7. 
Department of Mathematics,

Catholic University of Daefu,

Gyeongsan, Gyeongbuk, 713-702, Korea

E-mail: jykim@cu.ac.kr

Department of Mathematics,

Chosun University,

Kwangju, 501-759, S.Korea.

E-mail: sakim@mail.chosun.ac.kr 